\title{
A primary ion pump: proton/sodium pumping pyrophosphatases
}

\author{
Yuh-Ju Sun ${ }^{1}$, Kun-Mou Li ${ }^{1}$, Craig Wilkinson ${ }^{2}$ \& Adrian Goldman ${ }^{2,3}$ \\ 1 Institute of Bioinformatics and Structural Biology, College of Life Science, National Tsing Hua University, Hsinchu 30013, Taiwan. \\ 2 Astbury Centre for Structural Molecular Biology, School of Biomedical Sciences, University of Leeds, Leeds LS2 9JT, England. \\ 3 Division of Biochemistry, Department of Biosciences, University of Helsinki, Helsinki, FIN-00014, Finland.
}

Membrane bound pyrophosphatases (M-PPase) possess the enzymatic function to catalyse the hydrolysis of pyrophosphate (PPi) and show the ion pumping activity to transport ion across the membranes. M-PPase was isolated as a homodimer and each monomer contains 16 transmembrane helices. M-PPase from plant, Vigna radiate ( $V r P P a s e)$, function as a proton pump. However, M-PPase from bacterial, Thermotoga maritima (TmPPase), act as a sodium pump. The overall crystal structures of M-PPase folded in a rosette manner with two concentric walls, inner and outer walls. From M-PPase structures of various catalytic states, a complete catalytic cycle for M-PPases was proposed. The helical movements are found to involve in the closure and open of the substrate-binding pocket and to rearrange the key residues and leads to ion pumping. The structural and functional information provide the basis for understanding a unique ion translocation pathway and to identify the ion selection among various M-PPases. TmPPase and $V r$ PPase suggest a conserved coupling mechanism for M-PPase. 